\title{
WFR-2D: An Analytical Model for PWAS-Generated 2-D Ultrasonic Guided Wave Propagation
}

\author{
Yanfeng Shen, Victor Giurgiutiu \\ Department of Mechanical Engineering, University of South Carolina, Columbia, SC, 29208, USA
}

\begin{abstract}
This paper presents WaveFormRevealer 2-D (WFR-2D), an analytical predictive tool for the simulation of 2-D ultrasonic guided wave propagation and interaction with damage. The design of structural health monitoring (SHM) systems and self-aware smart structures requires the exploration of a wide range of parameters to achieve best detection and quantification of certain types of damage. Such need for parameter exploration on sensor dimension, location, guided wave characteristics (mode type, frequency, wavelength, etc.) can be best satisfied with analytical models which are fast and efficient.

The analytical model was constructed based on the exact 2-D Lamb wave solution using Bessel and Hankel functions. Damage effects were inserted in the model by considering the damage as a secondary wave source with complex-valued directivity scattering coefficients containing both amplitude and phase information from wave-damage interaction. The analytical procedure was coded with MATLAB, and a predictive simulation tool called WaveFormRevealer 2-D was developed. The wave-damage interaction coefficients (WDICs) were extracted from harmonic analysis of local finite element model (FEM) with artificial non-reflective boundaries (NRB). The WFR-2D analytical simulation results were compared and verified with full scale multiphysics finite element models and experiments with scanning laser vibrometer. First, Lamb wave propagation in a pristine aluminum plate was simulated with WFR-2D, compared with finite element results, and verified by experiments. Then, an inhomogeneity was machined into the plate to represent damage. Analytical modeling was carried out, and verified by finite element simulation and experiments. This paper finishes with conclusions and suggestions for future work.
\end{abstract}

Keywords: smart structure, structural health monitoring, nondestructive evaluation, guided waves, Lamb waves, analytical model, damage detection, piezoelectric wafer active sensors

\section{INTRODUCTION}

Self-sensing smart structures would be able to sense their environment while being aware of their own structural integrity. This paper focuses on the latter, i.e., the development of structural health monitoring (SHM) concepts and methods through predictive simulation and experimental verification. SHM systems aim at providing on-demand damage detection and real-time diagnosis of structural status. The development of computation models for Lamb wave propagation and interaction with damage is of great importance for both SHM system design and signal interpretation purposes. Effective design of SHM systems requires the exploration of a wide range of parameters (transducer size, sensor-damage relative locations, interrogating wave characteristics, etc.) to achieve best detection and quantification of certain types of damage. On the other hand, active sensing signals using Lamb waves are usually hard to interpret due to the multi-mode, dispersive nature of Lamb waves and their interaction with damage, involving complicated scattering and mode conversion phenomena. Practical applications impose three main requirements on computation models: (1) accuracy for high frequency, short wavelength, and long propagation distance waves; (2) efficiency in terms of computation time and computer resource; (3) versatility with a wide range of parameter exploration capability. The best choice for achieving parameter exploration is an analytical model, which allows the modification of calculation parameters with high computation efficiency.

\subsection{Piezoelectric wafer active sensors}

Piezoelectric wafer active sensors (PWAS) are convenient enablers for generating and receiving Lamb waves. They couple the electrical and mechanical effects (mechanical strain, $S_{i j}$, mechanical stress, $T_{k l}$, electrical field, $E_{k}$, and electrical displacement, $D_{j}$ ) through the tensorial piezoelectric constitutive equations

Health Monitoring of Structural and Biological Systems 2014, edited by Tribikram Kundu, Proc. of SPIE Vol. 9064,906411 .

(c) 2014 SPIE $\cdot$ CCC code: $0277-786 X / 14 / \$ 18 \cdot$ doi: 10.1117/12.2044798 


$$
\begin{aligned}
& S_{i j}=s_{i j k l}^{E} T_{k l}+d_{k i j} E_{k} \\
& D_{j}=d_{j k l} T_{k l}+\varepsilon_{j k}^{T} E_{k}
\end{aligned}
$$

where $s_{i j k l}^{E}$ is the mechanical compliance of the material measured at zero electric field $(E=0), \varepsilon_{j k}^{T}$ is the dielectric permittivity measured at zero mechanical stress $(T=0)$, and $d_{j k l}$ represents the piezoelectric coupling effect. PWAS utilize the $d_{31}$ coupling between in-plane strains, $S_{1}, S_{2}$ and transverse electric field $E_{3}$.

Compared with conventional ultrasonic transducers, PWAS are small, lightweight, low cost, and unobtrusive. They can be permanently bonded on host structures and achieve real time active sensing and in-situ monitoring. PWAS can serve several purposes ${ }^{1}$ : (a) high-bandwidth strain sensors; (b) high-bandwidth wave exciters and receivers; (c) resonators; (d) embedded modal sensors with the electromechanical (E/M) impedance method. By application types, PWAS transducers can be used for (i) active sensing of far-field damage using pulse-echo, pitch-catch, and phased-array methods, (ii) active sensing of near field damage using high-frequency E/M impedance method and thickness gage mode, and (iii) passive sensing of damage-generating events through detection of low-velocity impacts and acoustic emission at the tip of advancing cracks.

\subsection{State of the art}

Pioneer researches have been conducted for analytical models of PWAS generated Lamb waves and the tuning effect between PWAS transducers and host structures ${ }^{1,2,3}$. However, these models only consider the wave propagation in pristine structures, whereas the design of SHM systems requires wave-damage interaction also be studied.

Regarding the aspect of wave-damage interaction, considerable progresses have been achieved through analytical approaches. Some have used Kirchhoff, Mindlin, or Kane-Mindlin plate theory, ${ }^{4,5}$, while others proposed 3-D elasticity solution or exact Lamb mode solutions ${ }^{7,8}$, But these models only apply to simple damage geometries such as circular holes, partial-through circular holes, or flat-bottom cavities. Besides, these analytical solutions are usually mathematically complicated and hard to attain. Numerical methods, such as finite element method (FEM), boundary element method (BEM), spectral element method (SEM), and finite difference method (LISA) provide an alternative approach for attacking the wave scatter problem in a robust manner for complex damage geometries $9,10,11,12,13,14$. However, high frequency, short wavelength simulations require considerably small time marching step and dense discretization of the analysis domain, which can make the target problem computationally prohibitive. Semi-analytical methods and small-size numerical methods such as distributed point source method (DPSM) and semi-analytical finite element (SAFE) method have been developed to make the computation load manageable ${ }^{15,16}$. These methods either require evaluation of Green's function or further finite element formulation, where the user-friendliness and readiness remain to be improved for their applications.

This paper presents an analytical predictive tool, WaveFormRevealer 2-D (WFR-2D), which couples with local finite element models, for efficient simulation of Lamb wave propagation and interaction with damage. Wave generation, propagation, interaction with damage and detection are described using WFR-2D analytical framework, while wavedamage interaction coefficients (WDICs) are extracted from a local small-size FEM with non-reflective boundaries (NRB). WFR-2D can be used to explore a wide range of design parameters, as well as model different damage types with complicated geometries.

\section{2-D ANALYTICAL FRAMEWORK FOR SHM ACTIVE SENSING}

In this section, we will present the methodology of modeling damage effects, and detailed steps to construct the analytical framework for PWAS-generated 2-D Lamb wave propagation and interaction with damage.

\subsection{Modeling of damage effects using complex-valued WDICs}

Under electrical excitation, the transmitter PWAS (T-PWAS) generates Lamb waves into the host structure. They propagate along the structure, interact with damage, undergo scattering, mode conversion, and are finally picked up by the scanning laser vibrometer, where the out-of-plane velocity of Lamb waves is measured. The sensing point signal is comprised of two parts: (1) direct incident waves from the T-PWAS; (2) scattered waves from the damage interaction. Damage is modeled as a secondary wave source. The total wave field $W_{\text {TOTAL }}$ is the superposition of the incident wave field $W_{I N}$ and the scattered wave field $W_{S C}$ from the damage. 


$$
W_{\text {TOTAL }}=W_{I N}+W_{S C}
$$

We used frequency and direction dependent complex-valued wave-damage interaction coefficients (WDICs) to model the scattering, and mode conversion phenomena of wave-damage interaction. These WDICs are capable of describing scattered wave amplitude, phase information, as well as their dependence on frequency and direction.

Our notations are as follows: we use two letters to describe the interaction phenomena, with the first letter denoting the incident wave type, and the second letter denoting the resulting wave type. For instance, SS (symmetric-symmetric) means the incident symmetric waves scattered as symmetric waves, while SA (symmetric-antisymmetric) means incident symmetric waves scattered and mode converted to antisymmetric waves. Thus the complex-valued WDIC $C_{S S}(\omega, \theta) \cdot e^{-i \varphi_{S S}(\omega, \theta)}$ denotes the scattered symmetric mode generated by incident symmetric mode with amplitude ratio $C_{S S}(\omega, \theta)$ and phase shift $\varphi_{S S}(\omega, \theta)$. Similarly, $C_{S A}(\omega, \theta) \cdot e^{-i \varphi_{S A}(\omega, \theta)}$ represents the scattered antisymmetric mode generated by incident symmetric mode with amplitude ratio $C_{S A}(\omega, \theta)$ and phase shift $\varphi_{S A}(\omega, \theta)$. $\omega$ is the wave component frequency. $\theta$ represents the scattering angle with respect to incident wave direction. For specific damage, the WDICs can be extracted from small-size FEM with NRB developed in this study.

\subsection{Analytical framework for 2-D Lamb wave propagation and interaction with damage}

The analytical framework was constructed in frequency domain based on the exact 2-D Lamb wave solution in the following steps:

1. Perform Fourier transform of excitation signal;

2. Calculation of structure transfer function;

3. Solve for the incident waves at sensing location and the incident waves at damage location;

4. Generation of scattered waves at damage location and transfer of scattered waves to sensing location;

5. Calculation of total wave field at sensing location;

6. Solve for out-of-plane particle motion at sensing location (for comparison with laser measurement);

7. Inverse Fourier transform of frequency domain signal to obtain time varying response at sensing location.

Details of each step are given next.

STEP 1: Perform Fourier transform of the time-domain excitation signal $V_{T}(t)$ to obtain the frequency domain excitation spectrum, $\tilde{V}_{T}(\omega)$.

STEP 2: Calculation of structure transfer function. Detailed analytical derivation of 2-D Lamb waves generated by a circular transmitter PWAS is given in ref 17,

$$
\begin{aligned}
& \left.u_{r}(r)\right|_{z=d}=-\pi i \frac{a^{2} \tau_{a}}{2 \mu} \sum_{\xi^{S}} \frac{J_{1}\left(\xi^{S} a\right) N_{S}\left(\xi^{S}\right)}{D_{S}^{\prime}\left(\xi^{S}\right)} H_{1}^{(1)}\left(\xi^{S} r\right) e^{-i \omega t}-\pi i \frac{a^{2} \tau_{a}}{2 \mu} \sum_{\xi^{A}} \frac{J_{1}\left(\xi^{A} a\right) N_{A}\left(\xi^{A}\right)}{D_{A}^{\prime}\left(\xi^{A}\right)} H_{1}^{(1)}\left(\xi^{A} r\right) e^{-i \omega t} \\
& N_{S}(\xi)=\xi \eta_{S}\left(\xi^{2}+\eta_{S}^{2}\right) \cos \eta_{P} d \cos \eta_{S} d ; \quad D_{S}=\left(\xi^{2}-\eta_{S}^{2}\right)^{2} \cos \eta_{P} d \sin \eta_{S} d+4 \xi^{2} \eta_{P} \eta_{S} \sin \eta_{P} d \cos \eta_{S} d \\
& N_{A}(\xi)=-\xi \eta_{S}\left(\xi^{2}+\eta_{S}^{2}\right) \sin \eta_{P} d \sin \eta_{S} d ; \quad D_{A}=\left(\xi^{2}-\eta_{S}^{2}\right)^{2} \sin \eta_{P} d \cos \eta_{S} d+4 \xi^{2} \eta_{P} \eta_{S} \cos \eta_{P} d \sin \eta_{S} d
\end{aligned}
$$

where $a$ is the radius of the circular PWAS, $d$ is half plate thickness, and $r$ is the distance between the point of interest and T-PWAS. $\tau_{a}$ represents shear stress between the transducer and host structure and $\mu$ denotes the shear modulus of the structure. $J_{1}$ is Bessel function of order one, which captures tuning effect between PWAS and the host structure. $H_{1}^{(1)}$ is the first kind Hankel function of order one, which represents an outward propagating 2-D wave field. $\xi$ is the frequency dependent wavenumber calculated from the Rayleigh-Lamb equation ${ }^{18}$ given in Eq. (5). 


$$
\frac{\tan \eta_{S} d}{\tan \eta_{P} d}=\left[\frac{-4 \eta_{P} \eta_{S} \xi^{2}}{\left(\xi^{2}-\eta_{S}^{2}\right)^{2}}\right]^{ \pm 1} ; \quad \eta_{P}^{2}=\frac{\omega^{2}}{c_{P}^{2}}-\xi^{2} ; \quad \eta_{S}^{2}=\frac{\omega^{2}}{c_{S}^{2}}-\xi^{2} ; \quad c_{P}=\sqrt{\frac{\lambda+2 \mu}{\rho}} ; \quad c_{S}=\sqrt{\frac{\mu}{\rho}} ;
$$

where +1 exponent corresponds to symmetric Lamb wave modes and -1 exponent corresponds to antisymmetric Lamb wave modes. $c_{P}$ and $c_{S}$ represent the pressure wave speed and shear wave speed; $\lambda$ is Lame's constant of the structural material; $\rho$ is the material density. The transfer function $\kappa_{P W A S}(\omega)$ in Eq. (6) converts the applied voltage $\tilde{V}_{T}(\omega)$ into the shear stress $\tau_{a}$, where $r(\omega)$ is the stiffness ratio between host structure and T-PWAS ${ }^{19}$.

$$
\kappa_{P W A S}(\omega)=\frac{d_{31}}{s_{11}^{E}} \frac{r(\omega)}{1-r(\omega)}
$$

Taking Fourier transform of Eq. (3), one can obtain the frequency domain structure transfer function for both symmetric and anti-symmetric modes. For each wave mode, the corresponding wavenumber $\xi$ should be chosen.

$$
G(\omega, r)=-\pi^{2} i \frac{a^{2} \kappa_{P W A S}(\omega)}{\mu} \sum_{\xi} \frac{J_{1}(\xi a) N_{S}(\xi)}{D_{S}^{\prime}(\xi)} H_{1}^{(1)}(\xi r)
$$

STEP 3: Multiply the structure transfer function by frequency-domain excitation signal to obtain the direct incident waves at sensing location, where the distance $R_{I N}$ from T-PWAS up to sensing location is used. Similarly, multiply the structure transfer function up to damage location by frequency-domain excitation signal to obtain the interrogating waves arriving at damage, where the distance $R_{D}$ from T-PWAS up to the damage location is used.

$$
u_{I N}\left(\omega, R_{I N}\right)=\tilde{V}_{T}(\omega) \cdot\left[G^{S}\left(\omega, R_{I N}\right)+G^{A}\left(\omega, R_{I N}\right)\right] ; \quad u_{D}\left(\omega, R_{D}\right)=\tilde{V}_{T}(\omega) \cdot\left[G^{S}\left(\omega, R_{D}\right)+G^{A}\left(\omega, R_{D}\right)\right]
$$

It can be noticed that the direct incident wave field is the superposition of symmetric and antisymmetric wave modes.

STEP 4: Scattered wave source at damage location is obtained by modifying incident waves at the damage with WDICs.

$$
u_{N}^{S}=C_{S S}(\omega, \theta) e^{-i \varphi_{S S}(\omega, \theta)} \cdot u_{D}^{S}+C_{A S}(\omega, \theta) e^{-i \varphi_{A S}(\omega, \theta)} \cdot u_{D}^{A} ; \quad u_{N}^{A}=C_{S A}(\omega, \theta) e^{-i \varphi_{S A}(\omega, \theta)} \cdot u_{D}^{S}+C_{A A}(\omega, \theta) e^{-i \varphi_{A A}(\omega, \theta)} \cdot u_{D}^{A}
$$

where $u_{N}^{S}$ and $u_{N}^{A}$ represent the damage generated $\mathrm{S} 0$ and $\mathrm{A} 0$ new wave sources respectively. These scattered waves are transferred from damage up to the sensing point. The 2-D Lamb wave field eradiating from a point source takes the following solution as given in ref 17,20

$$
u_{r}=\sum_{n=1}^{\infty} a_{n}(z) H_{1}^{(1)}\left(\xi_{n} r\right) e^{-i \omega t}
$$

where $a_{n}(z)$ is the thickness dependent modeshape of wave mode number $n$. Since the amplitude relationship between the interrogating waves and the scattered waves is enclosed in the WDICs, the transfer function from the damage up to the sensing point is simply $H_{1}^{(1)}\left(\xi R_{S C}\right)$, where $R_{S C}$ is the distance from the damage up to the sensing location. Thus, the scattered waves arriving at the sensing point can be calculated.

$$
u_{S C}^{S}=u_{N}^{S} H_{1}^{(1)}\left(\xi^{S} R_{S C}\right) ; \quad u_{S C}^{A}=u_{N}^{A} H_{1}^{(1)}\left(\xi^{A} R_{S C}\right)
$$

STEP 5: The total wave field at the sensing location is the superposition of the direct incident waves calculated from Eq. (8) and the scattered waves calculated from Eq. (11).

$$
u_{\text {TOTAL }}=u_{I N}+u_{S C}
$$

It should be noted that the total wave field obtained in Eq. (12) is the in-plane wave displacement. Since laser vibrometer measures out-of-plane particle velocity, we need to convert this in-plane wave motion into out-of-plane wave motion.

STEP 6: The out-of-plane displacement wave field can be obtained by the modeshape component ratio.

$$
u_{z}^{S}=u_{r}^{S} \frac{U_{z}^{S}(\omega, d)}{U_{r}^{S}(\omega, d)} ; \quad u_{z}^{A}=u_{r}^{A} \frac{U_{z}^{A}(\omega, d)}{U_{r}^{A}(\omega, d)}
$$


where $U_{r}^{S}, U_{z}^{S}, U_{r}^{A}, U_{z}^{A}$ are Lamb wave modeshape displacement components evaluated at the top surface of the structure. The modeshape solutions can be found in ref 1 . The out-of-plane velocity was calculated by differentiating the out-of-plane displacement with respect to time. Through this differentiation, the wave amplitude will be modified by a factor of $-i \omega$ as shown in Eq. (14).

$$
U=u \cdot e^{-i \omega t} ; \quad \frac{\partial U}{\partial t}=\frac{\partial\left(u \cdot e^{-i \omega t}\right)}{\partial t}=-i \omega \cdot U
$$

Thus, the solution for out-of-plane velocity in frequency domain can be obtained in the following form.

$$
\dot{u}_{z}\left(\omega, R_{D}, R_{S C}, \theta\right)=-i \omega \cdot u_{z}
$$

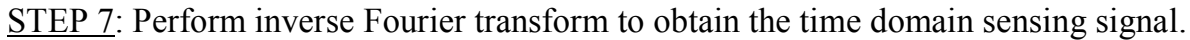

$$
\dot{u}_{z}\left(t, R_{D}, R_{S C}, \theta\right)=\operatorname{IFFT}\left[\dot{u}_{z}\left(\omega, R_{D}, R_{S C}, \theta\right)\right]
$$

In this study, we focus our attention on fundamental modes of Lamb waves ( $\mathrm{S} 0$ and $\mathrm{A} 0$ ), which find the widest application in Lamb wave based SHM. However, higher orders of Lamb modes (S1, A1, S2, A2, etc.) as well as shear horizontal ( $\mathrm{SH}$ ) modes from wave-damage interaction may also exist. The analytical framework can be easily extended to consider these aspects under the same principle. The difficulty may be found in the extraction of WDICs. The smallsize FEM technique developed in this study can solve the WDICs for S0, A0, SH0 wave modes. It should also be pointed out that, for model validation purpose, we used scanning laser vibrometer which measures the out-of-plane velocity of the wave field. Thus, the scattered SH waves, which do not have out-of-plane motion, cannot be captured.

\section{ANALYTICAL SIMULATION TOOL DEVELOPMENT: WFR-2D}

The analytical model is a general description of wave generation, propagation, damage interaction, and detection. Parameter exploration is made possible for T-PWAS size, structure material and thickness, sensor-damage locations, and arbitrary excitations, etc. This analytical framework was coded with MATLAB, and a graphical user interface (GUI) called WaveFormRevealer 2-D (WFR-2D) was developed. Figure 1 shows the WFR-2D interfaces.

(a) WFR Main Interface

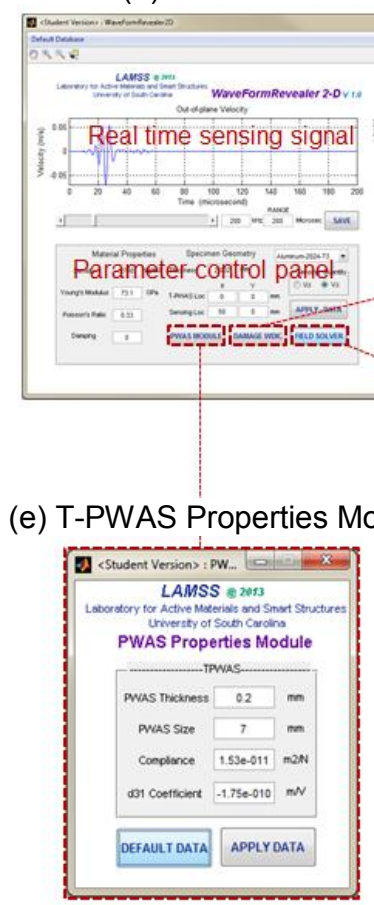

(b) Scatter Information Platform

(c) SO WDICs Module
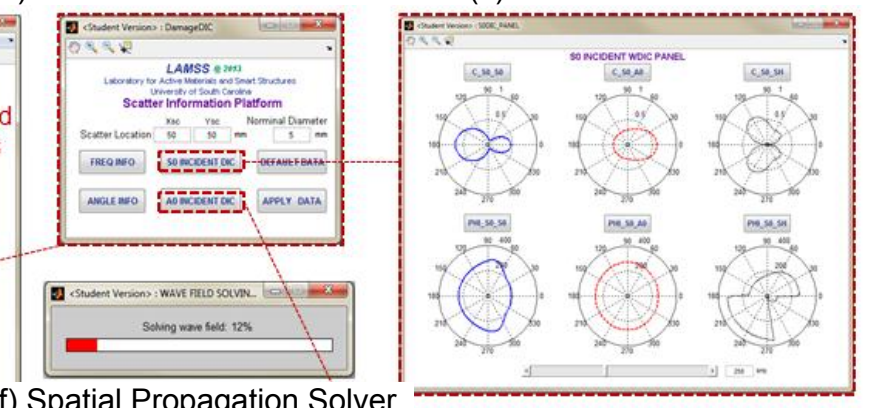

(d) AO WDICs Module

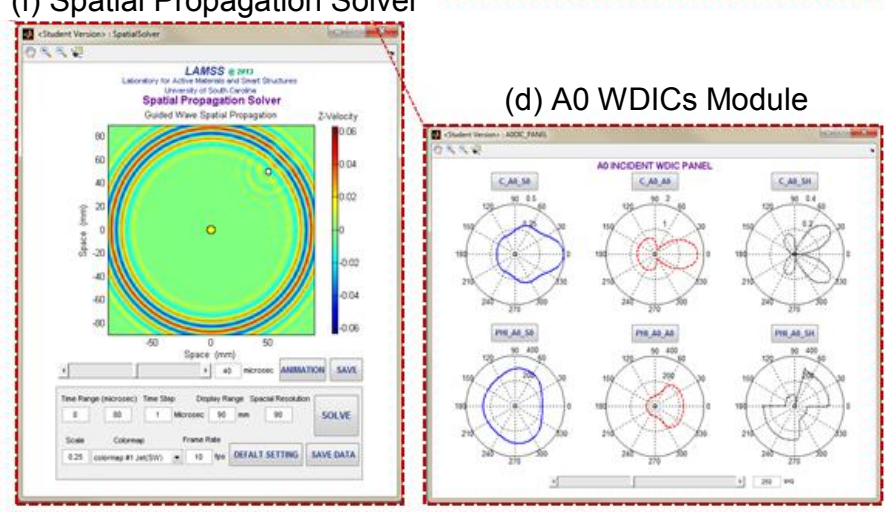

Figure 1: GUI of WFR-2D: (a) WFR-2D main interface; (b) damage information platform; (c) S0 WDICs module; (d) A0 WDICs module; (e) T-PWAS properties module; (f) spatial propagation solver 
Figure 1a shows the WFR-2D main interface which calculates the real time sensing signals as well as dispersion curves and tuning curves. The parameter control panel allows users to modify host structure material properties, thickness, and transmitter-damage-sensing locations. The excitation control panel provides excitation waveform, frequency, and arbitrary excitation loading options. Users can also selectively choose the excited wave mode of interest. Figure $1 \mathrm{~b}$ shows the scatter information platform for wave-damage interaction coefficients (WDICs) input. Figure 1c and Figure $1 \mathrm{~d}$ shows the sub-interfaces for loading S0 and A0 WDICs. The T-PWAS properties module allows users to define TPWAS geometric and material properties (Figure 1e). The spatial propagation solver, shown in Figure 1f, is like a Cscan, which calculates the transient time-space domain wave field. Thus the transient spatial wave field can be obtained at any instance during wave propagation. Users can visualize wave propagation and interaction with damage easily with the animation functions offered by WFR. Additionally, all the calculation data is available for user analysis with the data saving functions. With this analytical tool, considerable computation efficiency for large parameter space exploration can be achieved. It may take tens of hours for commercial finite element software to run a full scale 3-D simulation for an acceptable-accuracy solution of high frequency, short wavelength, and long distance wave problem; but it takes only several seconds to obtain the same solution with the WFR coupling with one or two hours local FEM calculation.

\section{SMALL-SIZE FINITE ELEMENT MODEL FOR THE EXTRACTION OF WAVE- DAMAGE INTERACTION COEFFICIENTS}

Figure 2 shows the schematic for WFR coupling with small-size FEM. Lamb wave generation, propagation, damage interaction (scattering, mode conversion), and detection are modeled using WFR, whereas the WDICs are extracted from harmonic analysis of small-size FEM with non-reflective boundaries (NRB). In this section, we will present the theory and methodology of WDIC extraction.

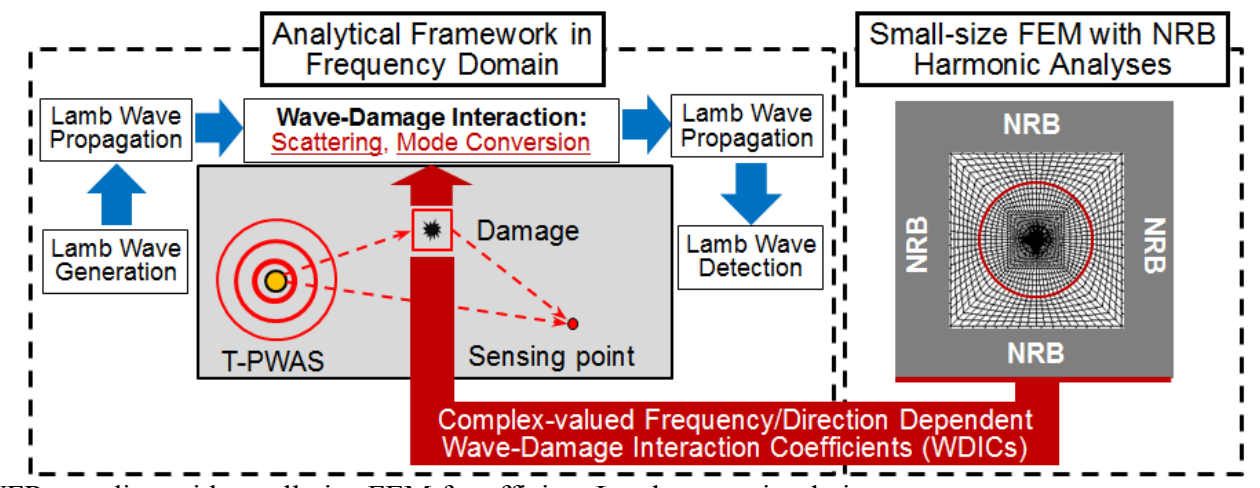

Figure 2: WFR coupling with small-size FEM for efficient Lamb wave simulation

\subsection{Harmonic analysis of small-size FEM with NRB}

We used COMBIN14 spring-damper elements to construct the NRB condition (for details of the NRB, readers are referred to ref 21). The use of NRB in FEM harmonic analysis permits the simulation of continuous harmonic wave incidence to interested region and its scatter from arbitrary damage under a specific frequency. Thus, the steady-state amplitude and phase information facilitate the extraction of WDICs $C_{\mathrm{AB}}(\omega, \theta) \cdot e^{-i \varphi_{\mathrm{AB}}(\omega, \theta)}$. In order to extract WDICs for an individual Lamb mode, a pair of harmonic analyses needs to be conducted: a pristine case and a damaged case. Figure 3 shows the small-size FEM pair designed for 2.032-mm thick 2024-T3 aluminum plate. Each model is $100 \mathrm{~mm}$ long, $100 \mathrm{~mm}$ wide, and $2.032 \mathrm{~mm}$ thick, with $30 \mathrm{~mm}$ wide NRB covering each boundary.

Figure 3 also shows the loading nodes and the sensing nodes. The loading nodes are aligned to create a straight crested harmonic incident wave field, which is a good approximation when the damage is located far away from the excitation source. In the pristine model, a circle of sensing nodes and one center sensing point are designed to collect structural harmonic response. The center sensing point records the incident waves arriving at damage location. In the damaged case, only a circle of sensing nodes, with the same location as the pristine model, are used to collect the response data. The design of circular positioned sensing nodes allows us to extract information for all the directions of interest. 
A damage example is shown in Figure 3. The geometry of damage is kept simple to ensure consistent FEM and experimental models for validation purpose, but complex enough to represent general wave-damage interaction phenomena, containing all the mode conversion possibilities. It should be noted that different types of damage will have different scattering characteristics and require a corresponding local damage model for WDICs extraction.
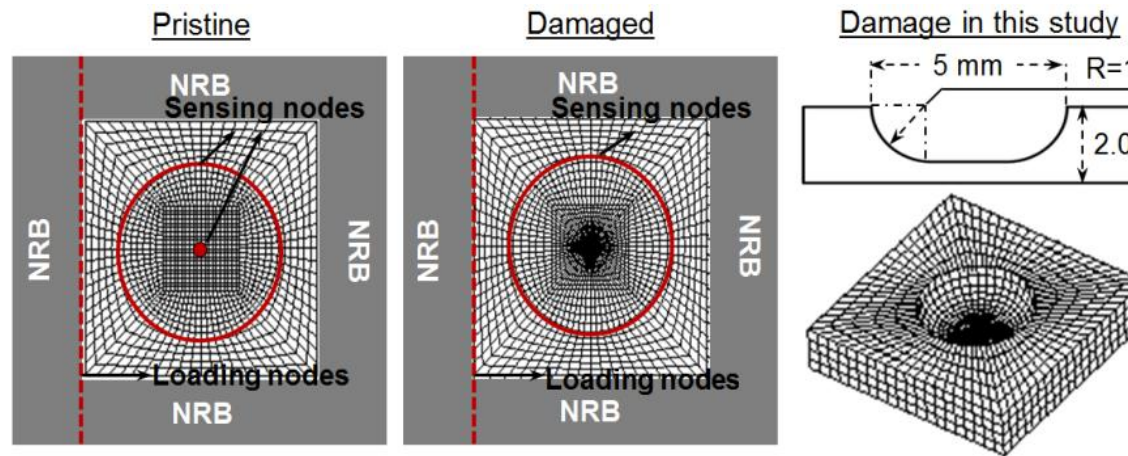

Figure 3: Small-size FEM for WDICs extraction

\subsection{WDICs extraction procedure}

Figure 4 shows the schematic of the sensing region for the small-size FEM for WDICs extraction. The in-plane displacements at the sensing nodes are used. According to Eq.(2), the scattered wave field can be obtained by subtracting the incident waves from the total waves.

$$
W_{S C}=W_{\text {TOTAL }}-W_{I N}
$$

The sensing nodes data in the pristine model is the incident wave field, while the data from damaged model represents the total wave field containing both incident and scattered waves. Thus, the subtraction of the data between these two models provides the scattered wave field in all directions. Using Eq. (17) and coordinate transformation, we calculated the scattered wave displacements at the top and bottom surface sensing nodes in both radial $\left(u_{r}^{T}\right.$ and $\left.u_{r}^{B}\right)$ and tangential $\left(u_{\theta}^{T}\right.$ and $u_{\theta}^{B}$ ) directions. Using Eq. (18), we can separate and selectively represent each wave mode.

$$
u_{S C}^{S 0}=\frac{u_{r}^{T}+u_{r}^{B}}{2} ; \quad u_{S C}^{A 0}=\frac{u_{r}^{T}-u_{r}^{B}}{2} ; \quad u_{S C}^{S H 0}=\frac{u_{\theta}^{T}+u_{\theta}^{B}}{2} ;
$$

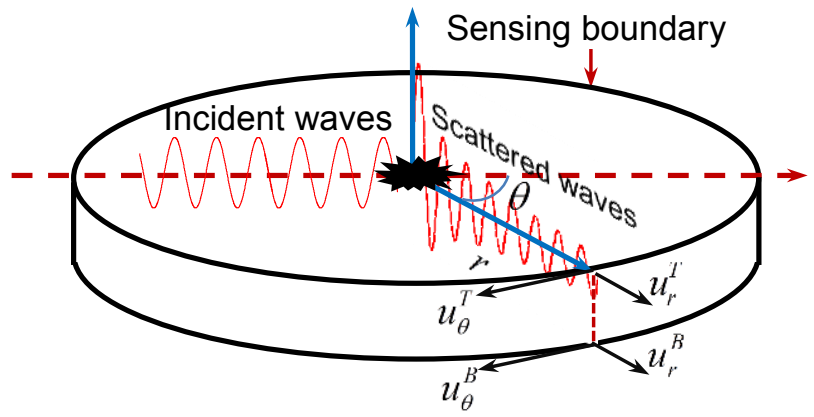

Figure 4: Extraction of WDICs from small-size FEM

The incident wave arriving at the damage location is recorded by the center sensing point and denoted as $u_{I N}$. The relationship between incident wave arriving at the damage and the scattered wave picked up on the sensing boundary can be formulated as Eq.(19).

$$
u_{I N}^{\mathrm{A}} e^{-i \varphi_{I N}^{\mathrm{A}}} \cdot C_{\mathrm{AB}}(\omega, \theta) e^{-i \varphi_{\mathrm{AB}}(\omega, \theta)} \cdot H_{m}^{(1)}\left(\xi^{\mathrm{B}} r\right)=u_{S C}^{\mathrm{B}}(\theta) e^{-i \varphi_{S C}^{\mathrm{B}}(\theta)}
$$

where $u_{I N}^{\mathrm{A}} e^{-i \varphi_{I N}^{\mathrm{A}}}$ is the $A$ mode incident wave recorded by the center sensing node with amplitude and phase information; $C_{\mathrm{AB}}(\omega, \theta) e^{-i \varphi_{\mathrm{AB}}(\omega, \theta)}$ is the WDICs, containing mode conversion ( $A$ mode to $B$ mode), direction dependency, amplitude 
ratio, and phase relationship information; $H_{m}^{(1)}\left(\xi^{\mathrm{B}} r\right)$ represents the outward propagating 2-D wave field of the resulting scattered wave mode $B$, with $m=1$ for Lamb waves and $m=0$ for SH waves. $u_{S C}^{\mathrm{B}}(\theta) e^{-i \phi_{S C}^{\mathrm{B}}(\theta)}$ is the scattered waves recorded on the sensing boundary with scatter angle $\theta$, amplitude and phase information.

The harmonic analysis provides the incident and scattered wave amplitude-phase information in Eq. (19), with $C_{\mathrm{AB}}(\omega, \theta) e^{-i \varphi_{\mathrm{AB}}(\omega, \theta)}$ left as the only unknown term. Upon rearrangement, Eq. (19) becomes

$$
C_{\mathrm{AB}}(\omega, \theta) e^{-i \varphi_{\mathrm{AB}}(\omega, \theta)}=\frac{u_{S C}^{\mathrm{B}}(\theta)}{u_{I N}^{\mathrm{A}}} \frac{1}{H_{m}^{(1)}\left(\xi^{\mathrm{B}} r\right)} e^{-\Delta \varphi_{\mathrm{AB}}(\theta)} ; \Delta \varphi_{\mathrm{AB}}(\theta)=\varphi_{S C}^{\mathrm{B}}(\theta)-\varphi_{I N}^{\mathrm{A}}
$$

By identification, the amplitude coefficient and phase coefficient can be extracted

$$
C_{\mathrm{AB}}(\omega, \theta)=\left|\frac{u_{S C}^{\mathrm{B}}(\theta)}{u_{I N}^{\mathrm{A}}} \frac{1}{H_{m}^{(1)}\left(\xi^{\mathrm{B}} r\right)}\right| ; \quad \varphi_{\mathrm{AB}}(\omega, \theta)=\Delta \varphi_{\mathrm{AB}}(\theta)-\left[\angle \frac{1}{H_{m}^{(1)}\left(\xi^{\mathrm{B}} r\right)}-\angle \frac{1}{H_{m}^{(1)}\left(0^{+}\right)}\right]
$$

\subsection{WDICs extraction results}

Figure 5 shows the WDICs of incident S0 waves at $200 \mathrm{kHz}$. It can be observed that the interaction between the incident S0 wave and damage not only involves scattered S0 waves, but also mode converted A0 and SH0 waves. These amplitude and phase coefficients are direction-dependent, which may indicate the best detection direction for certain wave mode as well as the damage. The calculation of amplitude coefficients has been investigated in many publications with various methods ${ }^{4,8,22}$, but the estimation of phase coefficients has not been discussed elsewhere. The phase coefficients are of great significance in simulating wave-damage interaction, because they govern the constructive or destructive superposition of incident and scattered wave fields. Figure 6 shows the WDICs of incident A0 wave at 200 $\mathrm{kHz}$. Similar scattering, mode conversion and direction-dependency phenomena are noticed in the coefficient patterns.
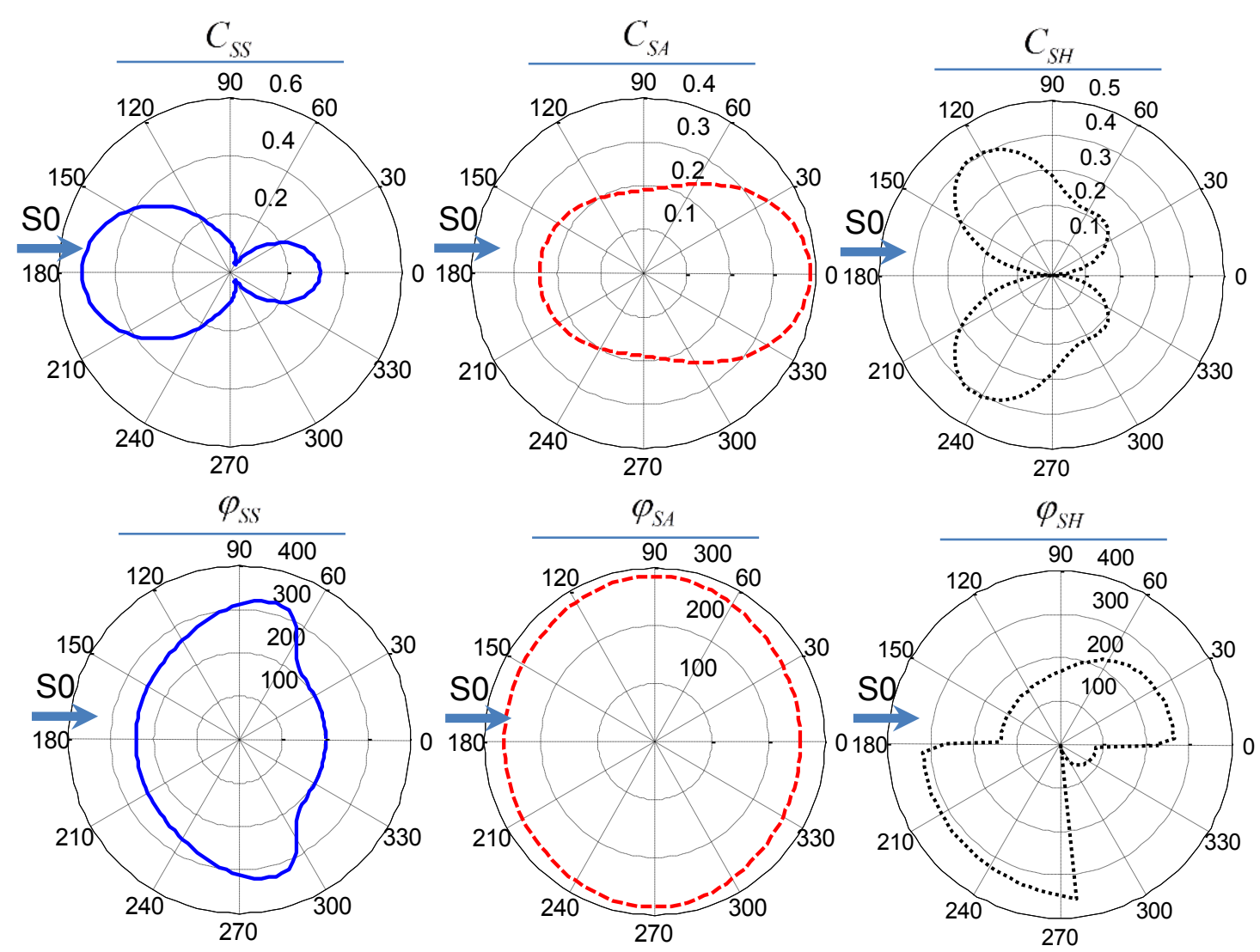

Figure 5: WDICs from $\mathrm{S} 0$ wave interaction at $200 \mathrm{kHz}$ 

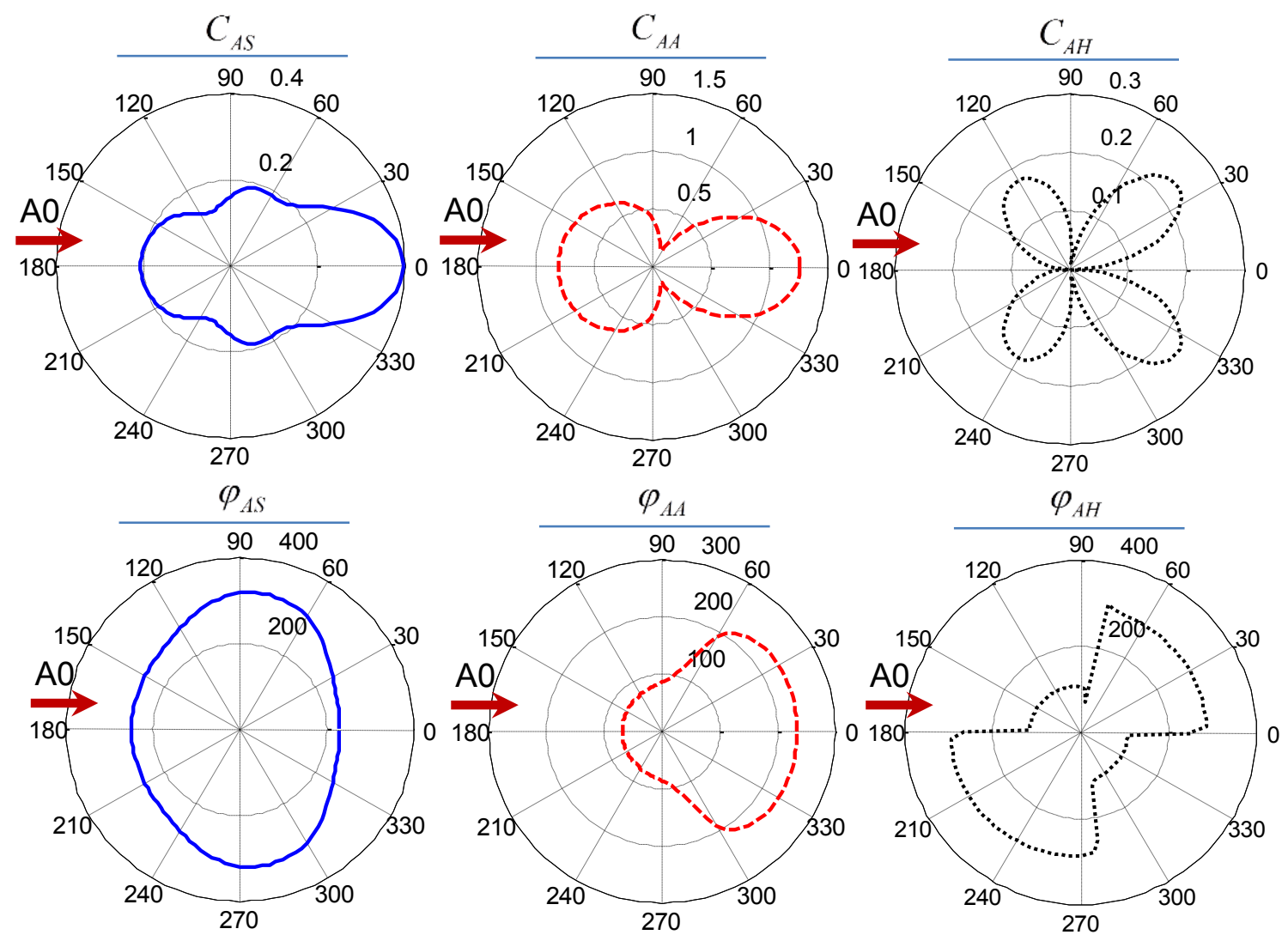

Figure 6: WDICs from A0 wave interaction at $200 \mathrm{kHz}$

\section{NUMERICAL AND EXPERIMENTAL VERIFICATIONS}

In this section, we will present the multiphysics finite element models and experiments for WFR verification. The comparison results are shown for wave propagation in a pristine plate and a damaged plate.

\subsection{Multi-physics finite element model and experimental setup}

Figure 7 shows the finite element model. The specimen is a $2.032 \mathrm{~mm}$ thick aluminum plate. To minimize the calculation burden, NRB were implemented around the model. We used SOLID5 coupled field elements to simulate the piezoelectric effect of T-PWAS, SOLID45 eight node structure element to mesh the plate, and COMBIN14 springdamper element to construct the NRB. The mesh size adopted in this study is $1 \mathrm{~mm}$ for in-plane direction and $0.5 \mathrm{~mm}$ for thickness direction. The T-PWAS and damage regions were meshed with even smaller elements to accommodate high stress gradient. A total of 423,468 elements were used. The time step was set to $0.25 \mu$ s. Two sets of simulations were carried out: (1) 2-D Lamb wave propagation in a pristine plate; (2) 2-D Lamb wave propagation in a damaged plate. The location and geometry information of the T-PWAS, damage and specimen are shown in Figure 7.
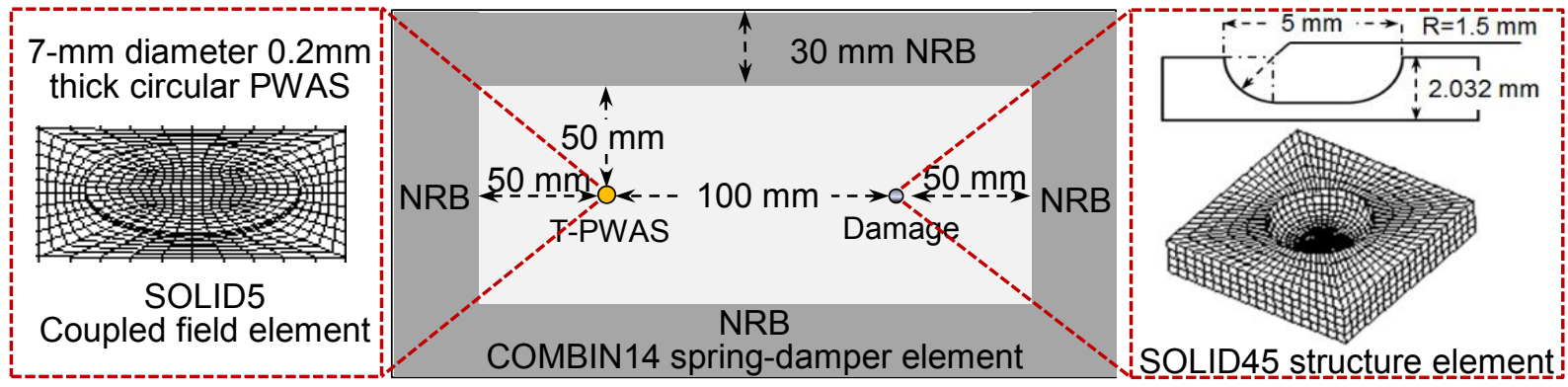

Figure 7: Multi-physics finite element model 
Figure 8 shows the experimental setup. The function generator was used to generate 3-count Hanning window modulated tone bursts which are amplified to $50 \mathrm{vpp}$ by the amplifier and applied on the T-PWAS. Lamb waves generated by T-PWAS propagated along the structure, interacted with the damage, and were measured by a Polytec PSV-400 scanning laser vibrometer. Reflective tape was used to enhance surface reflections and improve visualization quality. A C-scan of the specimen surface was carried out for both the pristine and the damaged plates. The locations of the T-PWAS, damage and special recording points are illustrated in Figure 8.

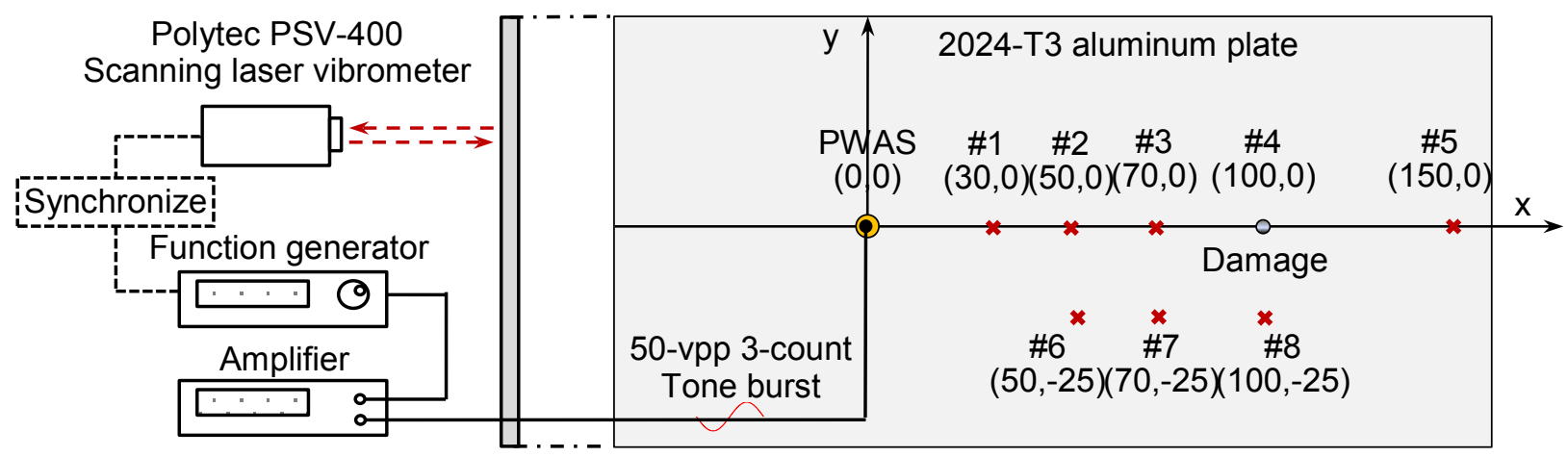

Figure 8: Experimental setup with scanning laser vibrometer

\subsection{Results and discussion}

\subsubsection{Lamb wave propagation in pristine plate}

Figure 9 shows the comparison of the transient spatial wave field among WFR-2D, FEM simulation, and experimental measurement for a pristine plate. It can be observed that WFR-2D predictions have good agreement with both FEM and experiment. A circular wave front was generated by the T-PWAS, strong near the wave source, and weak at far field due to the outward propagation pattern.

(a)
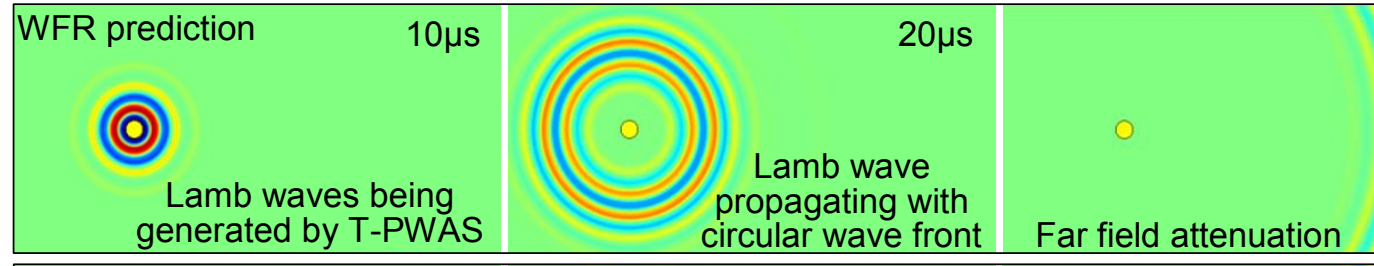

(b)

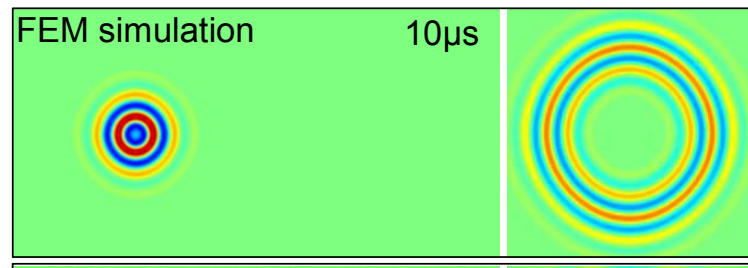

$20 \mu \mathrm{s}$

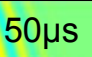

(c)
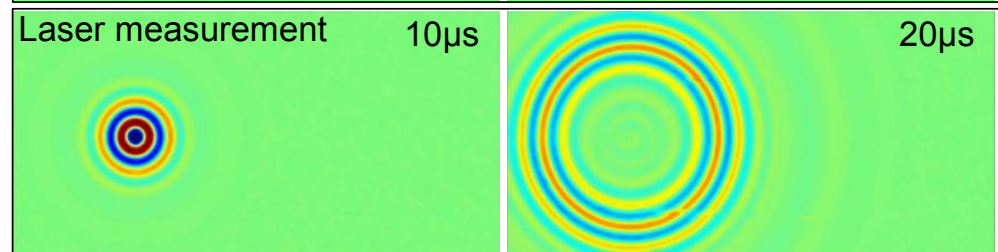

Figure 9: Comparison of $200 \mathrm{kHz}$ wave field in pristine plate: (a) WFR prediction; (b) FEM simulation; (3) experiment

Figure 10 shows the waveform validation results at various sensing locations for $200 \mathrm{kHz}$ excitation. It can be observed that WFR predictions have good match with both FEM simulation and experiments. At the near field (location \#2), S0 and $\mathrm{A} 0$ waves are mixed together. The FEM result shows a shift from the WFR prediction, while WFR prediction agrees well with laser measurement. It seems the FEM mesh adopted in this study does not capture the near field waves as accurate as WFR. WFR prediction of far field waveform agrees well with both FEM and experiments for a pristine plate. 


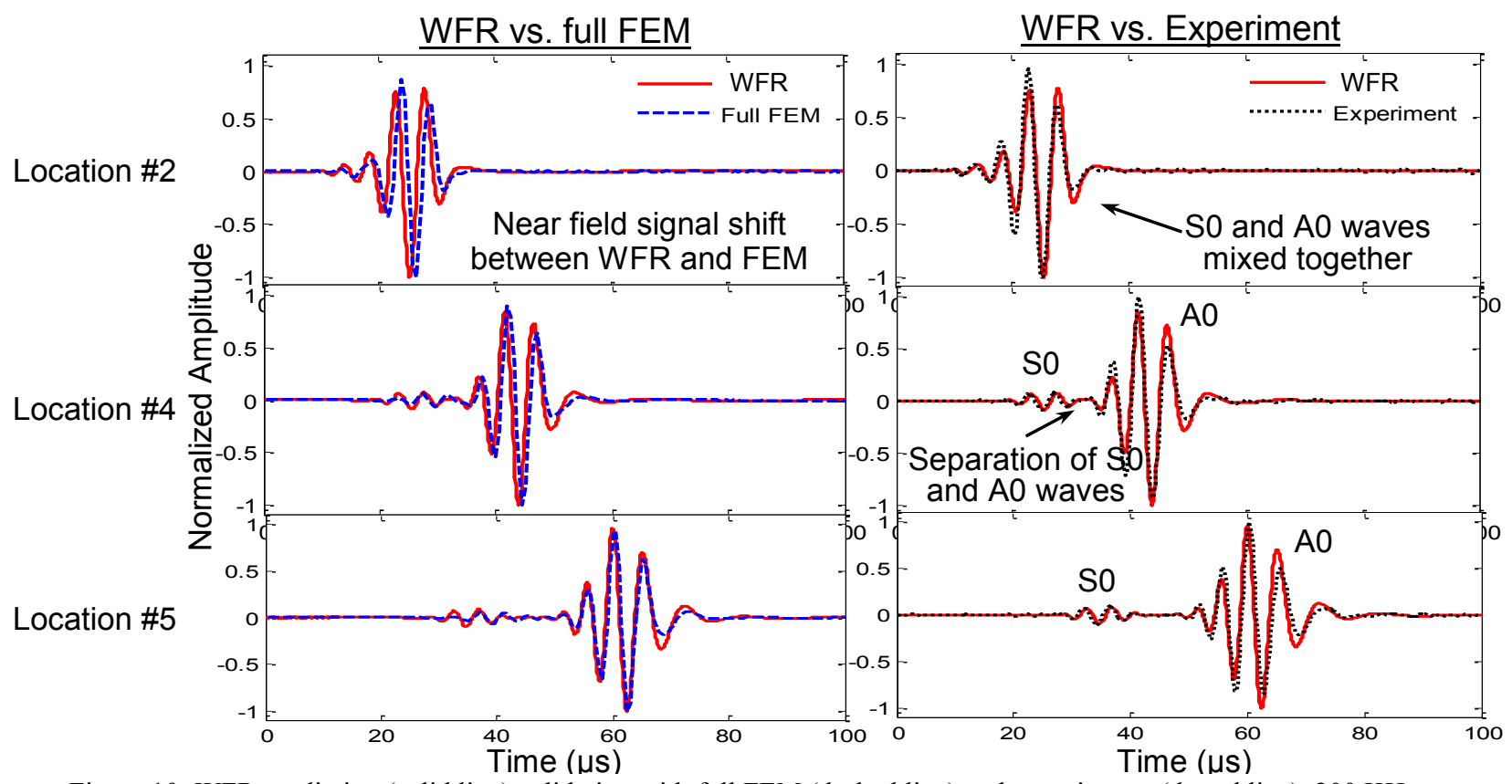

Figure 10: WFR prediction (solid line) validation with full FEM (dashed line) and experiments (dotted line). $200 \mathrm{KHz}$ signals of pristine plate at various sensing locations shown in Figure 8.

\subsubsection{Lamb wave propagation in damaged plate}

Figure 11 shows the comparison of transient spatial wave field among WFR-2D, full FEM simulation and experiment for damaged plate. It can be observed that WFR results agree well with FEM simulation and experimental measurement.

(a) WFR prediction

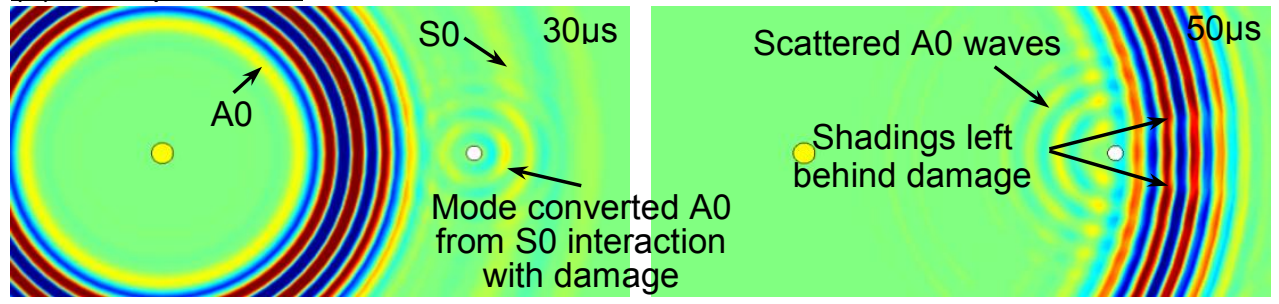

\section{(b) FEM simulation}

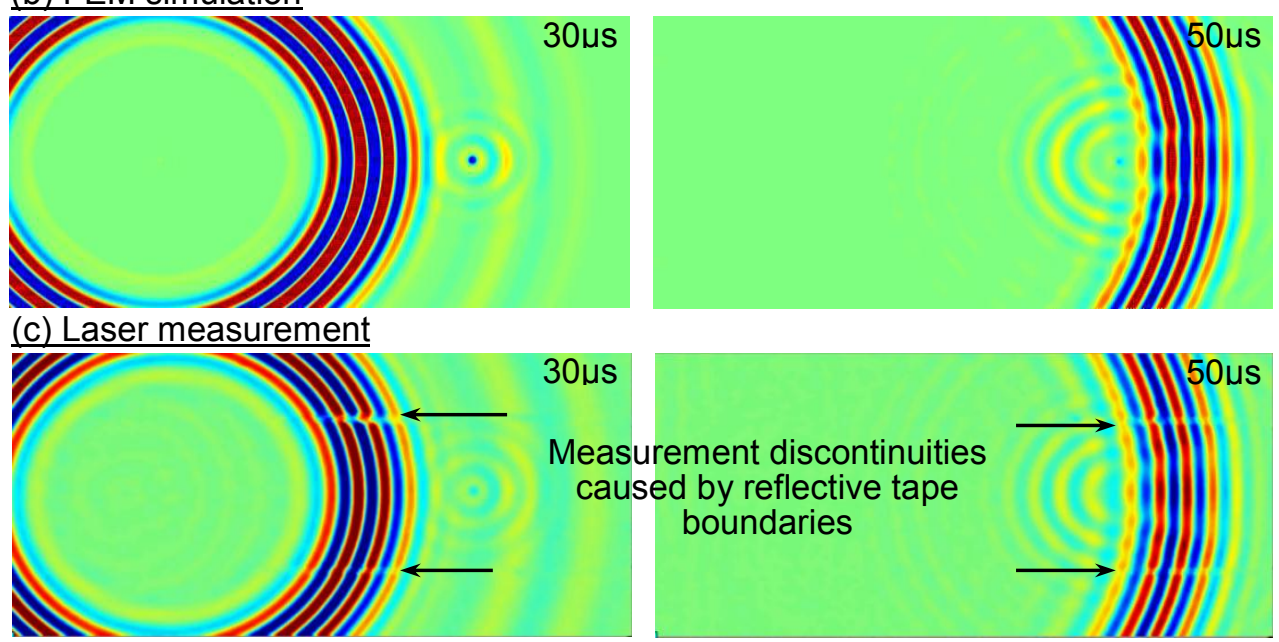

Figure 11: Comparison of wave field in damaged plate, showing S0 and A0 Lamb modes interacting with damage: (a) WFR prediction; (b) FEM simulation; (c) experiment 
At $30 \mu \mathrm{s}$, fast propagating S0 mode with long wavelength and slowly propagating A0 mode with short wavelength can be clearly identified. The mode converted A0 waves from S0 interaction with damage can be noticed, propagating with a short wavelength. At $50 \mu \mathrm{s}$, after A0 waves interact with damage, the scattered A0 mode can be observed as well as the shading left behind the damage. This shading effect is caused by the destructive superposition of incident and scattered A0 waves. This also illustrates the importance of obtaining phase information in the WDICs. It should be noted that measurement discontinuities were found in the experimental data, which were caused by the reflective tape boundaries.

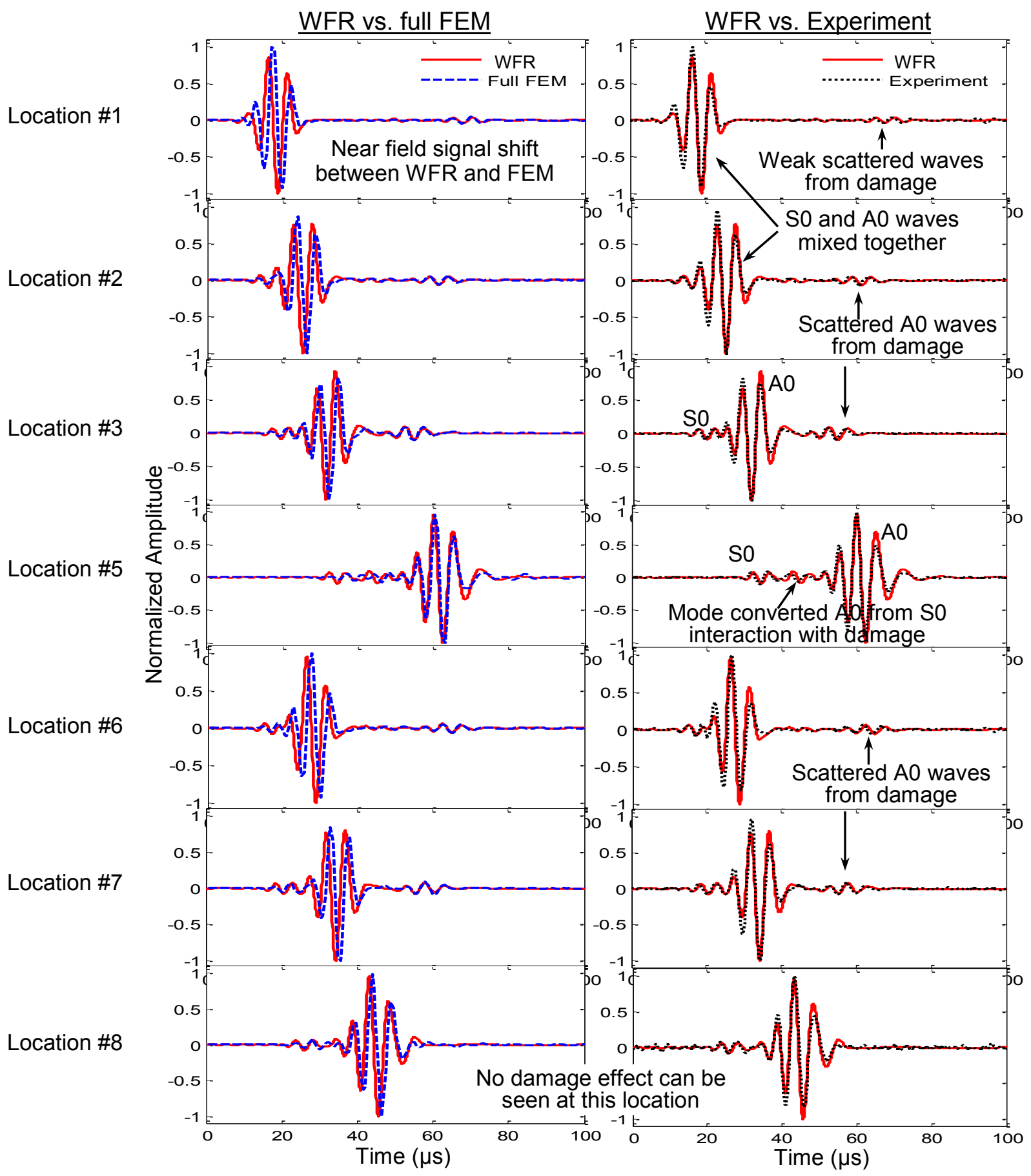

Figure 12: WFR prediction (solid line) validation with full FEM (dashed line) and experiments (dotted line). $200 \mathrm{KHz}$ signals of damaged plate at locations \#1 through \#8 shown in Figure 8. 
Figure 12 shows the waveform validation results at various sensing locations for $200 \mathrm{kHz}$ excitation. It can be observed that WFR predictions match well with FEM simulation and experiments. The signals at location \#1, \#2, and \#3 show that the scattered A0 wave amplitude increases when the sensing location moves closer to the damage. The signal at location \#5 shows the mode converted A0 wave packet from the S0 interaction with damage. Locations \#6, \#7, and \#8 are in certain directions with respect to the incident waves and damage. It should be noted that at location \# 6 and \#7, damage scattered waves can be observed, but at location \#8, no damage effect can be seen. Recall the WDICs pattern in Figure 6, the scattered A0 amplitude coefficient reaches a minimum value near 90 degrees with respect to the incident waves. This fact illustrates that at certain sensing location, the damage cannot be effectively detected. Using WFR-2D, the waveform can be predicted at arbitrary sensing locations, and the users can determine whether these locations are sensitive points or blind zones, optimizing the design of a sensor network for damage detection.

\section{CONCLUSIONS AND FUTURE WORK}

The WaveFormRevealer 2-D (WFR-2D) is a predictive tool for efficient simulation of 2-D Lamb wave propagation and interaction with damage. WFR-2D uses the exact 2-D Bessel function solution to calculate Lamb wave propagation excited by a piezo wafer and wave-damage interaction coefficients (WDICs) for scattered field prediction. The WDICs are calculated from an efficient local area finite element method (FEM) analysis. The WFR-2D results have been verified against full-scale multi-physics FEM simulations and scanning laser vibrometer experiments. Our WFR-2D has the following advantages over other SHM simulation tools:

1 Our WFR approach can deal with transient wave interaction with complicated damage type and geometries in an easier and more user-friendly way, than other analytical or semi-analytical formulations such as distributed point source method (DPSM) and semi-analytical finite element (SAFE) method ${ }^{15,16}$.

2 WFR does not require meshing of the entire structure, thus it minimizes the computation burden; WFR is constructed in frequency domain, and does not require time marching procedure, thus it saves considerable computation time, compared with conventional numerical methods such as finite element method (FEM) ${ }^{10}$, spectral element method (SEM) ${ }^{12}$, or finite difference method (LISA) ${ }^{13}$.

3 The harmonic analyses of small-size FEM with non-reflective boundaries (NRB) are faster and more target-oriented compared with conventional transient analyses. The use of NRB minimizes the model size and eliminates redundant boundary reflections. Also, harmonic analysis does not require the results of previous calculation step to solve the current step as it is the case in conventional transient analysis.

4 Because it is fast and efficient, WFR-2D can be used for parameter studies of various engineering scenarios. It enables users to define transducer size, structure material and thickness, sensor-damage locations, and arbitrary excitations by interacting with the graphical user interface. This user-friendly feature and parameter definition freedom allows users to explore the best sensor arrangement for damage detection in a highly efficient manner, whereas any of these parameter changes will result in re-built/re-mesh/re-run of the whole model in the case of conventional FEM simulation.

\subsection{Original contributions}

1 We have developed a novel approach to coupling analytical model with local FEM for simulation of 2-D interaction between guided waves and damage.

2 We have introduced the complex-valued WDICs which are capable of describing mode conversion, amplitude, phase, and directionality information during wave damage interaction. We have found that the phase information is very important, although it was ignored by previous researchers.

3 Our method utilizes small-size local FEM harmonic analysis with NRB to determine the WDICs over the frequency band required for analytical simulation.

4 The WFR-2D graphical user interface provides a versatile user-friendly capability for studying a large family of SHM problems.

\subsection{Future work}

Verification of other types of damage with experiments should be performed. Extension to more than the basic S0, A0, and SH0 modes should be made. Inclusion of nonlinear effects should be attempted. WFR concept should be developed 
to simulate wave propagation in composite structures. The small-size FEM should be further explored to obtain characteristics of waves excited by arbitrary shaped PWAS transducers on various kinds of structures.

\section{ACKNOWLEDGEMENTS}

Support from Office of Naval Research \# N00014-11-1-0271, Dr. Ignacio Perez, Technical Representative; Air Force Office of Scientific Research \#FA9550-11-1-0133, Dr. David Stargel, Program Manager; SPARC (Support to Promote Advancement of Research and Creativity) at University of South Carolina; are thankfully acknowledged.

\section{REFERENCES}

[1] V. Giurgiutiu, [Structural healthing monitoring with piezoelectric wafer active sensors], Elsevier Academic Press, (2007).

[2] V. Giurgiutiu, "Tuned Lamb wave excitation and detection with piezoelectric wafer active sensors for structural health monitoring," Journal of intelligent material systems and structures, pp. 291-306, (2005).

[3] A. Raghavan and C. E. S. Cesnik, "Finite-dimensional piezoelectric transducer modeling for guided wave based structural health monitoring," Smart Materials and Structures, vol. 14, pp. 1448-1461, (2005).

[4] C. Vemula and A. N. Norris, "Flexual wave propagation and scattering on thin plates using Mindlin theory," Wave Motion, vol. 26, pp. 1-12, (1997).

[5] J. C. P. McKeon and M. K. Hinders, "Lamb waves scattering from a through hole," Journal of Sound and Vibration, pp. 843-862, (1999).

[6] C. Wang and F. Chang, "Scattering of plate waves bya cylindrical inhomogeneity," Journal of Sound and Vibration, vol. 282, pp. 429-451, (2005).

[7] T. Grahn, "Lamb wave scattering from a circular partly through-thickness hole in a plate," Wave Motion, (2002).

[8] L. Moreau, M. Caleap, A. Velichko and P. D. Wilcox, "Scattering of guided waves by flat-bottomed cavities with irregular shapes," Wave Motion, p. doi: 10.1016/j.wavemoti.2011.12.004, (2012).

[9] D. Alleyne and P. Cawley, "The interaction fo Lamb waves with defects," IEEE Transactions on Ultrasonics Ferroelectrics and Frequency Control, vol. 39, no. 3, pp. 381-397, (1992).

[10] Z. Chang and A. Mal, "Scattering of Lamb waves from a rivet hole with edge cracks," Mechanics of Materials, pp. 197-204, (1999).

[11] Y. Cho, D. Hongerholt and J. Rose, "Estimation of ultrasonic guided wave mode conversion in a plate with thickness variation," IEEE Transaction on Ultrasonics, Ferroelectrics, and Frequency Control, vol. 47, no. 3, (2000).

[12] W. Ostachowicz, P. Kudela, M. Krawczuk and A. Zak, [Guided Waves in Structures for SHM: The Time-Domain Spectral Element Method], West Sussex, UK: Wiley, (2012).

[13] K. Nadella and C. Cesnik, "Local interaction simulation approach for modeling wave propagation in composite structures," CEAS Aeronaut Journal, vol. 4, pp. 35-48, (2013).

[14] Y. Shen and V. Giurgiutiu, "Predictive modeling of nonlinear wave propagation for structural health monitoring with piezoelectric wafer active sensors," Journal of Intelligent Material Systems and Structures, vol. 25, no. 4, pp. 506-520, (2014).

[15] E. Rahani and T. Kundu, "Modeling of Transient Ultrasonic Wave Propagation Using the Distributed Point Source Method," IEEE Transactions on Ultrasonics, Ferroelectrics, and Frequency Control, vol. 58, no. 10, (2011).

[16] A. Srivastava, [Quantitative structural health monitoring using ultrasonic guided waves], $\mathrm{PhD}$ Thesis, University of California, San Diego, CA, (2009).

[17] V. Giurgiutiu, [Structural Health Monitoring with Piezoelectric Wafer Active Sensors], Second Edition, Elsevier Academic Press, (2014).

[18] K. F. Graff, [Wave motion in elastic solids], New York: Dover publications, INC., (1991). 
[19] B. Lin, A. Kamal, V. Giurgiutiu and T. Kamas, "Multimodal Lamb Waves Power and Transfer Function Analysis of Structurally-bounded PWAS," in ASME 2012 SMASIS, Sone Mountain, Geogia, USA, (2012).

[20] E. Glushkov, N. Glushkova, R. Lammering, A. Eremin and M. Neumann, "Lamb wave excitation and propagation in elastic plates with surface obstacles: proper choice of central frequency," Smart Materials and Structures, (2011).

[21] Y. Shen, [Structural Health Monitoring Using Linear and Nonlinear Ultrasonic Guided Waves], Ph.D. Dissertation, University of South Carolina, (2014).

[22] L. Moreau, A. Velichko and P. Wilcox, "Accurate finite element modeling of guided wave scattering from irregular defects," NDT\&E International, vol. 45, pp. 46-54, (2012). 